\title{
Analytical prediction of friction factors and Nusselt numbers of turbulent forced convection in rod bundles with smooth and rough surfaces
}

\author{
Jian $\mathrm{Su}^{\mathrm{a}, *, 1}$, Atila P. Silva Freire ${ }^{\mathrm{b}}$ \\ a Nuclear Engineering Program, COPPE, Universidade Federal do Rio de Janeiro, C.P. 68509, 21945-970 Rio de Janeiro, Brazil \\ ${ }^{\mathrm{b}}$ Mechanical Engineering Program, COPPE, Universidade Federal do Rio de Janeiro, C.P. 68503, \\ 21945-970 Rio de Janeiro, Brazil
}

Received 15 March 2001; received in revised form 1 February 2002; accepted 7 April 2002

\begin{abstract}
A simple analytical method was developed for the prediction of the friction factor, $f$, of fully developed turbulent flow and the Nusselt number, $N u$, of fully developed turbulent forced convection in rod bundles arranged in square or hexagonal arrays. The friction factor equation for smooth rod bundles was presented in a form similar to the friction factor equation for turbulent flow in a circular pipe. An explicit equation for the Nusselt number of turbulent forced convection in rod bundles with smooth surface was developed. In addition, we extended the analysis to rod bundles with rough surface and provided a method for the prediction of the friction factor and the Nusselt number. The method was based on the law of the wall for velocity and the law of the wall for the temperature, which were integrated over the entire flow area to yield algebraic equations for the prediction of $f$ and $N u$. The present method is applicable to infinite rod bundles in square and hexagonal arrays with low pitch to rod diameter ratio, $P / D<1.2$. (C) 2002 Elsevier Science B.V. All rights reserved.
\end{abstract}

\section{Introduction}

Accurate knowledge of the friction factor of turbulent flow and the Nusselt number of turbu-

\footnotetext{
* Corresponding author. On leave at: Department of Chemical Engineering and Chemical Technology, Imperial College of Science and Technology, London SW7 2BY, UK. Tel.: +44-20-7589-5111x55671; fax: + 44-20-7594-5604

E-mail address: sujian@lmn.con.ufrj.br (J. Su).

${ }^{1}$ Tel.: +55-21-2562-8410; fax: +55-21-2562-8444; s.jian@ic.ac.uk.
}

lent forced convection in rod bundles in square or hexagonal arrays is essential for the design and reliable operation of nuclear reactors and is important also in other engineering applications. A large number of experimental and theoretical works have been done in the last 50 years, but accurate and reliable correlations for the friction factor and the Nusselt number in such a complex turbulent flow are still to be achieved.

The purpose of this work is to develop a simple analytical method for the prediction of the friction factor, $f$, of fully developed turbulent flow 
and the Nusselt number, $N u$, of fully developed turbulent forced convection in rod bundles arranged in square or hexagonal arrays. For the friction factor in rod bundles with smooth surface, we followed the work of Lee (1995), but presented the friction factor equation in a different form, which is similar to the friction factor equation for turbulent flow in a circular pipe. We applied the same analytical method to derive an explicit equation for the Nusselt number of turbulent forced convection in rod bundles with smooth surface. In addition, we extended the analysis to rod bundles with rough surface and provided a method for the prediction of the friction factor and Nusselt number. The prediction of the friction factor and Nusselt number was based on the law of the wall for velocity and the law of the wall for temperature which were integrated over the entire flow area to yield algebraic equations for the prediction of $f$ and $N u$. The laws of the wall for velocity and temperature assume the same classical forms as for turbulent flow in a circular pipe. We used two empirical expressions, one for the variation of the local friction velocity and the other for the variation of the local friction temperature, to assess the behaviour of the flow parameters along the rod periphery. The present method is applicable to infinite rod bundles in square and hexagonal arrays with low pitch-to-diameter ratio, $P / D<1.2$.

Before moving to the analytical development, we first present a literature survey of related works.

\section{Literature survey}

Many studies had been carried out in the 1970 s on turbulent flow and heat transfer in rod bundles on hexagonal arrays partly due to their importance in liquid-metal cooled fast breeder reactors (LMFBRs). Rehme (1972) investigated the pressure drop of rod bundles with pitch to rod diameter ratios $P / D=1.025-2.324$ and Reynolds numbers in the range 600-200000. He compiled experimental and theoretical results on pressure drop coefficients in rod bundles by over 60 authors and compared with his own results. Rehme
(1972) concluded that the pressure drop coefficients in closely packed rod bundles are considerably deviated from that for a circular pipe. In a subsequent paper, Rehme (1973) proposed a simple method for the prediction of the friction factor in non-circular channels, written as

$\sqrt{\frac{8}{\lambda}}=A\left[2.5 \ln \operatorname{Re} \sqrt{\frac{\lambda}{8}}+5.5\right]-G^{*}$

which furnishes good results provided the geometry factors for laminar now, $A$ and $G^{*}$, are known.

Trupp and Azad (1975) studied experimentally fully turbulent flow for three rod spacings $(P / D=$ $1.2,1.35$ and 1.5) over the Reynolds number range of 12000-84000 in a wind tunnel. They measured the local wall shear stress, the distribution of mean axial velocity, Reynolds stress and eddy diffusivities and discussed the structure of turbulent flow with the secondary flow pattern.

In search for a theoretical approach to the problem, Carajileskov and Todreas (1976) applied a one-equation turbulence model to compute the velocity field of axial and secondary flows in an interior subchannel of a bare rod bundle. They measured the distribution of axial velocity, turbulent kinetic energy and Reynolds stresses using a laser Doppler anemometer (LDA). This work was followed by a two-equation anisotropic turbulence modelling of the flow (Bartzis and Todreas, 1979). Heat transfer predictions were also made to confirm the importance of anisotropic viscosity in temperature predictions; these predictions, however, were not compared with experimental data. Parallelly, Fakory and Todreas (1979) investigated experimentally the wall shear stress distribution around the rod periphery, static pressure distribution, turbulence intensity and the friction factor in the central subchannel for Reynolds number between 4000 and 36000 and showed that the maximum wall shear stress occurs at the largest flow area and that the static pressure is not uniform around the rod periphery.

Turbulent flow and heat transfer in rod bundles in square arrays were also investigated extensively. Marek et al. (1973) covered results of pressure drop and heat transfer on two rod bundles with smooth surfaces; also pressure losses at 
the spacers and the circumferential temperature distribution were measured. They proposed relations for the friction factor and the Nusselt number in analogue to circular pipe correlations. Hooper (1980) measured the mean velocity variation, the wall shear stress variation and the six components of the symmetrical Reynolds stress tensor of turbulent flow in rod bundles in square arrays at two spacings $(P / D=1.194,1.107)$. The departure of the turbulent flow structure from the circular pipe flow was found to depend strongly on the $P / D$ ratio. In a follow up paper, Hooper and Wood (1984) used the axial momentum integral equation to show that the shear stress distribution is primarily determined by the pressure gradient and the shear stress $\overline{u^{\prime} v^{\prime}}$, a result that confirms the negligible influence of the mean secondary flow on flow parameters. In the same year, Hooper and Rehme (1984) showed that the axial and azimuthal turbulence intensities in the rod gap region increase strongly with decreasing rod spacing. The existence of features that promote intersubchannel momentum and heat transfer reduce the azimuthal variation of both the skin-friction coefficient and the heat transfer. The distribution of wall shear stress, mean axial velocities and turbulence intensities for the Reynolds number 500000 were measured by Renksizbulut and Hadaller (1986) through the laser Doppler anemometry and calibrated Preston tubes.

Cheng and Todreas (1985) presented a comprehensive study on hydrodynamic models and correlations for bundle friction factors, subchannel friction factors and mixing factors in bare and wire-wrapped hexagonal rod bundles. Vijayan et al. (1999) carried out experiment measurements of the pressure drop across various components of a pressurised heavy water reactor (PHWR) fuel channel under single-phase conditions. Recently, Chun and Seo (2001) performed an experimental study and a comparative study of five existing correlations for wire-wrapped fuel assemblies.

There had been more interests in the structure of turbulent flow and heat transfer in rod bundles in the 1980s and 1990s. Distributed parameter analysis demand empirical information on both turbulent momentum and energy transfer (Krauss and Meyer, 1998). A series of systematic experi- mental studies have been carried out at Kernforschungszentrum Karlsrushe (KfK) on turbulent flow through rod bundles and resulted in a large quantity of experimental data of the structure of turbulent flow and heat transfer. Rehme (1987) reviewed experimental investigations on the structure of turbulent flow through rod bundles and reported important results of a systematic experimental study of turbulent flow through subchannels of rod bundles showing clearly that turbulent flow through rod bundles differs greatly from turbulent flow through circular tubes. Rehme (1987) performed measurements of the mean velocity, wall shear stresses, and turbulence intensities in a rod bundle of four parallel rods arranged in a rectangular channel $(P / D=W / P=1.148)$ for three ratios of length-tohydraulic diameter $\left(L / D_{\mathrm{h}}\right)$. Rehme (1992) reviewed the experimental data on natural mixing between subchannels of rod bundles by turbulent exchange and concluded that cyclic and almost periodic flow pulsations through the gaps of rod bundles are the reason for the observed mixing rates through the gaps which are relatively independent of the gap width. Möller (1991) performed an experimental investigation on the turbulent flow in several rod bundles with different aspect rations. Hot wires and microphones were used for the measurements of velocity and wall pressure fluctuations. Based on the experimental results, a phenomenological model was proposed to explain the quasi-periodic behaviour of the turbulent fluctuating velocities in the axial and azimuthal directions. Subsequently, Möller (1992) investigated single-phase turbulent mixing in rod bundles by means of hot-wire measurements of spectra of the components of the turbulent fluctuating velocities in the gap regions of rod bundles and proposed a phenomenological model for the flow pulsation and mixing factors. Meyer (1994) investigated fully developed turbulent air flow in a heated 37-rod bundle with a pitch-to-diameter ratio of 1.12. Krauss and Meyer (1996) investigated turbulent air flow in a wall subchannel of a heated 37-rod bundle $(P / D=1.12, W /$ $D=1.06)$ by performing measurements with a hot-wire probe with $\mathrm{x}$-wires and a temperature wire. The mean velocity, mean fluid temperature, 
wall shear stress and wall temperature, turbulent quantities such as the turbulent kinetic energy, Reynolds stresses, and turbulent heat fluxes were measured. Krauss and Meyer (1998) investigated turbulent air flow in a central channel of heated 37-rod bundles with triangular array at two-different pitch-to-diameter ratios $(P / D=1.06$ and $P / D=1.12$ ). Time mean quantities and turbulent quantities were measured.

In the continual search for accurate correlations, Kim et al. (1993) developed an analytical model to predict the pressure drop and the heat transfer of turbulent flow in rod bundles. They divided the flow channel into a number of element channels and obtained the skin friction equation by integrating the logarithmic law of the wall in each element channel and summed up. The same was carried out for the Stanton number. However, this method cannot be applied to low pitchto-diameter ratio $(P / D<1.2)$ as the circumferential variation of local wall shear stress and temperature was not considered. Lee (1995) developed an analytical method for the prediction of the friction factor of turbulent flow in rod bundles of low pitch-to-diameter ratio by introducing the variation of local friction velocity along the rod periphery and integrating the law of the wall for velocity in whole flow area. The predicted friction factors are in good agreement with available experimental data. To our knowledge, no such a study has been done for the prediction of the Nusselt number in rod bundles of low pitch-to-diameter ratio.

We see through the literature survey that, as expected, the experimental and theoretical studies of turbulent flow and convection in rod bundles have been conducted always in comparison with the turbulent and convection in circular pipes. The correlations for the friction factor and the Nusselt number were usually presented as corrections to the circular pipe values at the same Reynolds and Prandtl numbers by multiplying a factor dependent on the pitch-to-diameter ratio. However, in a recent review on the prediction of turbulent flow and convection in round tubes, Churchill (2001) argued that the Nusselt number does not appear to be a fixed power of the tube Reynolds number and that the Nusselt number is found to be a fixed power of the Prandtl number only in the limit of infinity. He further pointed out that the use of the correlations of Nusselt number as a fixed power function of both Reynolds number and the Prandtl number has actually impeded the representation, understanding, and the prediction of turbulent convection.

\section{Analysis}

\subsection{Friction factor for rod bundles with smooth surface}

We follow the work of Lee (1995) in the development of an analytical prediction of the friction factor of turbulent flow in rod bundles with smooth surface, but present the friction factor equation in a different form, which is similar to the friction factor equation for turbulent flow in a circular pipe (White, 1974).

The law of the wall for incompressible turbulent flow over a smooth surface assumes the classical form

$\frac{u}{u_{\tau}}=\frac{1}{x} \ln \frac{y u_{\tau}}{v}+B$,

where $u_{\tau}$ is the friction velocity, $v$ is the kinematic viscosity, $x(=0.40)$ and $B(=5.5)$ are constants.

Following Lee (1995) we adopt the Fanning friction factor $f$, which is related to the friction velocity through the following expression

$\left(\frac{f}{2}\right)^{1 / 2}=\frac{\bar{u}_{\tau}}{u}$

where $\bar{u}$ is the mean velocity in the flow channel and $\bar{u}_{\tau}$ the mean friction velocity in the channel perimeter.

The friction factor equation in a circular pipe can be deduced by applying the law of the wall over the entire pipe radius. Integration of the classical law of the wall, Eq. (2), results in

$\left(\frac{2}{f}\right)^{1 / 2}=\frac{1}{\varkappa} \ln \left[\operatorname{Re}\left(\frac{f}{2}\right)^{1 / 2}\right]+B-\frac{3}{2 \varkappa}$,

where $R e$ is the Reynolds number based on the pipe diameter. 
For the typical geometry of an infinite rod bundle the mean velocity can evaluated through the integration

$$
\begin{aligned}
\bar{u} & =\frac{1}{A} \int u \mathrm{~d} A \\
& =\frac{1}{A} \int_{0}^{\theta_{\max }} \int_{0}^{(P / 2 \sec \theta-R)} u(y)(R+y) \mathrm{d} y \mathrm{~d} \theta
\end{aligned}
$$

where $P$ is the pitch of the rod bundle, and $R$ the radius of the rods. The distance $y$ is taken from the periphery of the rod outward in radial direction; the angle $\theta_{\max }$ defines the type of the rod bundle. For a rod bundle in hexagonal array. $\theta_{\max }=\pi / 6$; for a rod bundle in square array. $\theta_{\max }=\pi / 4$. Fig. 1 shows the typical geometry of infinite rod bundles in hexagonal or square arrays together with definition of the coordinate and parameters.

The area of the characteristic domain of the flow channel is obtained from the following integral

$A=\int_{0}^{\theta_{\max }} \int_{0}^{(P / 2 \sec \theta-R)}(R+y) \mathrm{d} y \mathrm{~d} \theta$.

Applying the law of the wall to the whole flow channel area $A$, we proceed to substitute Eq. (2) into Eq. (5) to find the friction factor equation. However, in closed packed rod bundles, the wall shear stress varies along the rod periphery, and thus, the integration cannot be performed unless this angular variation is modelled. Based on data of Trupp and Azad (1975), Fakory and Todreas (1979) for the hexagonal array and of Hooper (1980), Renksizbulut and Hadaller (1986) for the square array, it is possible to write

$u_{\tau}=\bar{u}_{\tau} F(\theta)$.

The function $F(\theta)$ is given by

$F(\theta)=\left(\frac{\tau_{w}}{\bar{\tau}_{w}}\right)^{1 / 2}=(1-a \cos 6 \theta-b \cos 12 \theta)^{1 / 2}$,

hexagonal array

$F(\theta)=\left(\frac{\tau_{w}}{\bar{\tau}_{w}}\right)^{1 / 2}=(1-a \cos 4 \theta-b \cos 8 \theta)^{1 / 2}$,

square array

where the constants $a$ and $b$ are shown in Table 1 , given by Lee (1995) through a data fitting.

We obtain the mean flow velocity by introducing the local variation of the friction velocity into the law of the wall and integrating over the channel area $A$

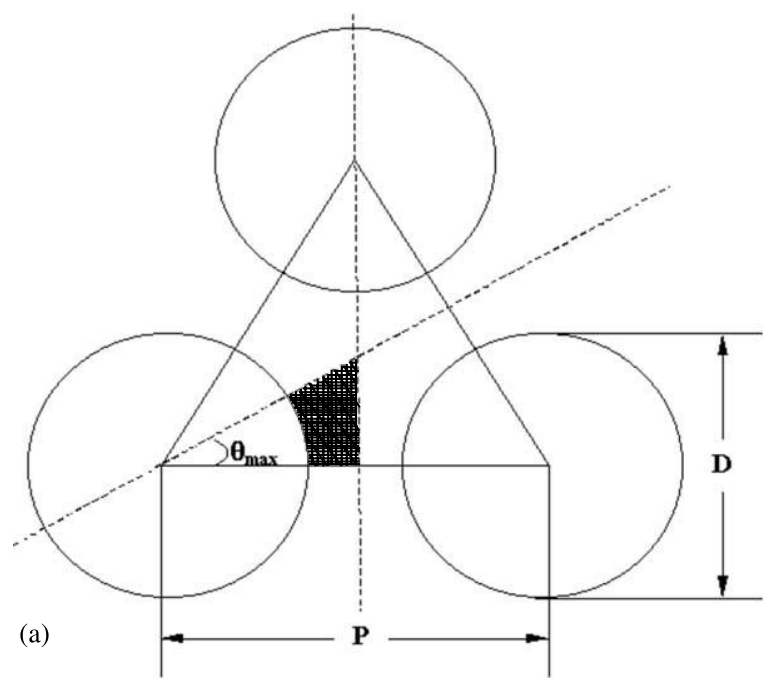

Fig. 1. Schematic diagram of rod bundles: (a) hexagonal array; (b) square array.

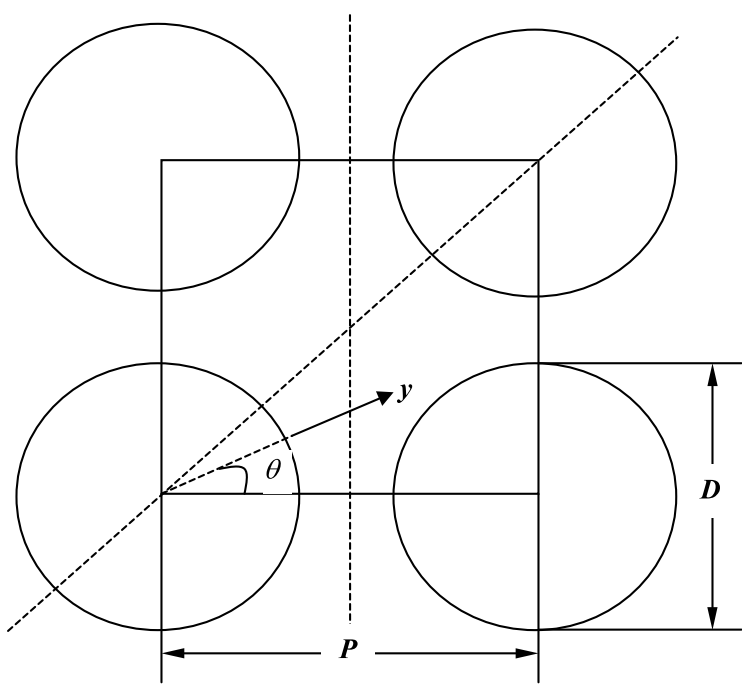

(b) 
Table 1

Constants $a$ and $b$

\begin{tabular}{llll}
\hline Hexagonal array & & & \\
$P / D$ & 1.05 & 1.10 & 1.20 \\
$a$ & 0.25 & 0.12 & 0.02 \\
$b$ & 0.02 & 0.02 & 0.01 \\
Square array & & & \\
$P / D$ & 1.11 & 1.15 & 1.19 \\
$a$ & 0.20 & 0.09 & 0.07 \\
$b$ & 0.02 & 0.02 & 0.02 \\
\hline
\end{tabular}

$\frac{\bar{u}}{\bar{u}_{\tau}}=\frac{1}{A} \int_{0}^{\theta_{\max }} \int_{0}^{(P / 2 \sec \theta-R)}\left(\frac{1}{\chi} \ln \frac{y \bar{u}_{\tau} F(\theta)}{v}+B\right) F(\theta)$

$\times(R+y) \mathrm{d} y \mathrm{~d} \theta$

which leads to the following equation for the mean friction velocity

$\frac{\bar{u}}{\bar{u}_{\tau}}=\left(\frac{1}{x} \ln \frac{D_{\mathrm{H}} \bar{u}_{\tau}}{v}+B\right) \alpha_{1}+\frac{\alpha_{2}}{x}$

where $\alpha_{1}$ and $\alpha_{2}$ are dimensionless geometrical parameters. The development of Eq. (10) and the definition of the parameters are given in the Appendix B.

Using the definition of the friction factor, Eq. (3), and of the Reynolds number based on the hydraulic diameter $D_{\mathrm{H}}, R e=D_{\mathrm{H}} \bar{u} / v$, we obtained the friction factor equation of turbulent flow in rod bundles with smooth surface

$\left(\frac{2}{f}\right)^{1 / 2}=\left(\frac{1}{x} \ln \left[\operatorname{Re}\left(\frac{f}{2}\right)^{1 / 2}\right]+B\right) \alpha_{1}+\frac{\alpha_{2}}{x}$.

3.2. Nusselt number for rod bundles with smooth surface

All the above ideas can be applied to the temperature field, provided the thermal law of the wall is considered together with the concept of bulk temperature.

Our objective is to determine the Nusselt number of turbulent forced convection, defined by

$N u=\frac{h D_{\mathrm{H}}}{\lambda}=\frac{\bar{q}_{\mathrm{w}} D_{\mathrm{H}}}{\lambda\left(\bar{T}_{\mathrm{w}}-\bar{T}\right)}$,

where $h$ is the heat transfer coefficient of turbulent forced convection, and $\lambda$ is the thermal conductivity of the fluid. The Nusselt number can be ob- tained if we first determine the Stanton number, defined by

$S t=\frac{\bar{q}_{\mathrm{w}}}{\rho c_{\mathrm{p}} \bar{u}\left(\bar{T}_{\mathrm{w}}-\bar{T}\right)}$

The thermal law of the wall for flow over a smooth surface can be written as (White, 1974)

$\frac{T_{\mathrm{w}}-T}{T_{\tau}}=\frac{1}{\varkappa} \ln \frac{y u_{\tau}}{v}+B_{\mathrm{t}}(P r)$,

where $T_{\mathrm{w}}$ is the wall temperature, $\kappa_{\mathrm{t}}(=0.44)$, and $B_{\mathrm{t}}$ is a function of the Prandtl number, $\mathrm{Pr}$, through equation

$B_{\mathrm{t}}(\operatorname{Pr})=13 \operatorname{Pr}^{2 / 3}-7$

The friction temperature $T_{\tau}$ is defined by

$T_{\tau}=\frac{q_{\mathrm{w}}}{\rho c_{\mathrm{p}} \bar{u}}$

It is easily seen that

$S t=\frac{\bar{u}_{\tau} \bar{T}_{\tau}}{\bar{u}\left(\bar{T}_{\mathrm{w}}-\bar{T}\right)}$.

The bulk temperature of the fluid is defined by

$\begin{aligned} \bar{T} & =\frac{\int T u \mathrm{~d} A}{\int u \mathrm{~d} A} \\ & =\frac{1}{\bar{u} A} \int_{0}^{\theta_{\max }} \int_{0}^{(P / 2 \sec \theta-R)} u(y) T(y)(R+y) \mathrm{d} y \mathrm{~d} \theta,\end{aligned}$

Similarly to the wall shear stress behaviour, we expect the wall heat transfer to vary along the rod periphery. Thus, if somehow this variation can be accounted for, then Eqs. (14) and (18) can be used to give predictions of the heat transfer coefficient.

Here, invoking the existing similarity between the transport mechanisms in the velocity and temperature fields for turbulent flows we make

$T_{\tau}=\bar{T}_{\tau} H(\theta), \quad$ and $\quad H(\theta)=F(\theta)$.

Applying the laws of the wall for velocity and temperature over the flow channel area $A$ we have 


$$
\begin{aligned}
\frac{1}{S t}= & \frac{\bar{u}\left(\bar{T}_{w}-\bar{T}\right)}{\bar{u}_{\tau} \bar{T}_{\tau}} \\
= & \frac{1}{A} \int_{0}^{\theta_{\max }} \int_{0}^{(P / 2 \sec \theta-R)}\left(\frac{1}{x} \ln \frac{y \bar{u}_{\tau} F(\theta)}{v}+B\right) \\
& \times\left(\frac{1}{x_{\mathrm{t}}} \ln \frac{y \bar{u}_{\tau} F(\theta)}{v}+B_{\mathrm{t}}(P r)\right) F(\theta) H(\theta)(R+y) \\
& \times \mathrm{d} y \mathrm{~d} \theta
\end{aligned}
$$

Further simplifying and using the definitions of the friction factor and the Reynolds number, we have

$$
\begin{aligned}
\frac{1}{S t}= & \beta_{0}\left(\ln \left[\operatorname{Re}\left(\frac{f}{2}\right)^{1 / 2}\right]\right)^{2} \\
& +\left(\beta_{1}+\gamma_{1} B_{\mathrm{t}}(\operatorname{Pr})\right) \ln \left[\operatorname{Re}\left(\frac{f}{2}\right)^{1 / 2}\right]+\beta_{2} \\
& +\gamma_{2} B_{\mathrm{t}}(\operatorname{Pr})
\end{aligned}
$$

The development of the equation and the definition of geometrical parameters above are given in the Appendix B. The Nusselt number is thus determined

$N u=S t R e P r=$

$\frac{\operatorname{RePr}}{\beta_{0}\left(\ln \left[\operatorname{Re}\left(\frac{f}{2}\right)^{1 / 2}\right]\right)^{2}+\left(\beta_{1}+\gamma_{1} B_{\mathrm{t}}(\operatorname{Pr})\right) \ln \left[\operatorname{Re}\left(\frac{f}{2}\right)^{1 / 2}\right]+\beta_{2}+\gamma_{2} B_{\mathrm{t}}(\operatorname{Pr})}$

\subsection{Rod bundles with rough surface}

Many specialisations of the law of the wall have been proposed in literature to account for such diverse effects as surface roughness, transpiration, pressure gradients, three-dimensionality, compressibility and shock-wave interaction, among many others. In this work, we are interested in flow over rough surface and will refrain ourselves by just introducing the formulation of Clauser (1956) for pipe flow, for the average roughness height denoted by $k$

$$
\frac{u}{u_{\tau}}=\frac{1}{\chi} \ln \frac{y u_{\tau}}{v}+B-\Delta B\left(k^{+}\right)
$$

$$
\Delta B_{\text {sand grains }}=\frac{1}{\varkappa} \ln \left(1+0.3 k^{+}\right),
$$

Three roughness regimes can be defined by using $k^{+}=k u_{\tau} / v$, i.e. hydraulically smooth wall for $k^{+}<4$, transitional-roughness regime for $4<$ $k^{+}<60$, and fully rough flow for $k^{+}>60$. In the last case, Clauser's law of wall, Eqs. (23) and (24) reduces to that proposed by Nikuradse (1933).

Parallel to the analysis of turbulent flow and heat transfer in rod bundles with smooth surface, we apply the law of the wall for velocity over rough surface, Eq. (23), in the flow channel and integrate the velocity distribution over the area $A$. We have then

$$
\begin{aligned}
\frac{\bar{u}}{\bar{u}_{\tau}}= & \frac{1}{A} \int_{0}^{\theta_{\max }} \int_{0}^{(P / 2 \sec \theta-R)}\left(\frac{1}{\varkappa} \ln \frac{y \bar{u}_{\tau} F(\theta)}{v}+B\right. \\
& \left.-\Delta B\left(k^{+}\right)\right) F(\theta)(R+y) \mathrm{d} y \mathrm{~d} \theta
\end{aligned}
$$

Using the analytical integrals in $y$ given in the Appendix B, we simplify Eq. (25) to

$$
\begin{aligned}
\frac{\bar{u}}{\bar{u}_{\tau}}= & \frac{1}{\alpha_{0}} \int_{0}^{\theta_{\max }}\left[\left(\frac{1}{\varkappa} \ln \frac{D_{\mathrm{H}} \bar{u}_{\tau} F(\theta)}{v}+B-\Delta B\left(k^{+}\right)\right) I_{0}(\theta)\right. \\
& \left.+\frac{I_{1}(\theta)}{\varkappa}\right] F(\theta) \mathrm{d} \theta
\end{aligned}
$$

which is a transcendental equation for the friction velocity $\bar{u}_{\tau}$. It can be readily solved by using standard root-finding methods combined with numerical integration for the evaluation of the righthand side of the equation. The friction factor is thus determined by Eq. (3).

Similarly, we apply the idea to the analysis of turbulent forced convection in rod bundles with rough surface. Applying the laws of the wall for velocity, Eq. (23), and for temperature Eq. (14), and integrating over the area $A$, we have

$$
\begin{aligned}
\frac{\bar{u}\left(\bar{T}_{\mathrm{w}}-\bar{T}\right)}{\bar{u}_{\tau} \bar{T}_{\tau}}= & \frac{1}{A} \int_{0}^{\theta_{\max }} \int_{0}^{(P / 2 \sec \theta-R)}\left(\frac{1}{\varkappa} \ln \frac{y \bar{u}_{\tau} F(\theta)}{v}+B\right. \\
& \left.-\Delta B\left(k^{-}\right)\right)\left(\frac{1}{\chi_{\mathrm{t}}} \ln \frac{y \bar{u}_{\tau} F(\theta)}{v}+B_{\mathrm{t}}(\operatorname{Pr})\right) \\
& \times F(\theta) H(\theta)(R+y) \mathrm{d} y \mathrm{~d} \theta,
\end{aligned}
$$

which is further simplified to an equation for the Stanton number by using the integrals defined in the Appendix B 


$$
\begin{aligned}
\frac{1}{S t}= & \frac{1}{\alpha_{0}} \int_{0}^{\theta_{\max }}\left[\left(\frac{1}{\varkappa} \ln \frac{D_{\mathrm{H}} \bar{u}_{\tau} F(\theta)}{v}+B-\Delta B\left(k^{+}\right)\right)\right. \\
& \times\left(\frac{1}{\chi_{\mathrm{t}}} \ln \frac{D_{\mathrm{H}} \bar{u}_{\tau} F(\theta)}{v}+B_{\mathrm{t}}(\operatorname{Pr})\right) I_{0}(\theta) \\
& +\left(\left(\frac{1}{\varkappa}+\frac{1}{\chi_{t}}\right) \ln \frac{D_{\mathrm{H}} \bar{u}_{\tau} F(\theta)}{v}+B-\Delta B\left(k^{+}\right)\right. \\
& \left.\left.+B_{\mathrm{t}}(\operatorname{Pr})\right) I_{1}(\theta)+\frac{I_{2}(\theta)}{\varkappa \varkappa_{t}}\right] F(\theta) H(\theta) \mathrm{d} \theta,
\end{aligned}
$$

As the friction velocity is already determined by using Eq. (26), we can see that Eq. (28) is an explicit equation for the Stanton number, which involves numerical integration for the right-hand side. The Nusselt number can now calculated, $N u=S t R e P r$.

Table 2

Geometrical parameters in the friction factor equation (Eq. (11)) for smooth rod bundles in hexagonal arrays

\begin{tabular}{lll}
\hline$P / D$ & $\alpha_{1}$ & $\alpha_{2}$ \\
\hline 1.10 & 1.01244 & -2.37478 \\
1.11 & 1.00989 & -2.38237 \\
1.12 & 1.00778 & -2.38909 \\
1.13 & 1.00604 & -2.39513 \\
1.14 & 1.00463 & -2.40064 \\
1.15 & 1.00351 & -2.40574 \\
1.16 & 1.00264 & -2.41053 \\
1.17 & 1.00201 & -2.41507 \\
1.18 & 1.00159 & -2.41941 \\
1.19 & 1.00137 & -2.42361 \\
1.20 & 1.00132 & -2.42769 \\
\hline
\end{tabular}

Table 3

Geometrical parameters in the friction factor equation (Eq. (11)) for smooth rod bundles in square arrays

\begin{tabular}{lll}
\hline$P / D$ & $\alpha_{1}$ & $\alpha_{2}$ \\
\hline 1.10 & 1.04356 & -2.30132 \\
1.11 & 1.03511 & -2.31324 \\
1.12 & 1.02802 & -2.32384 \\
1.13 & 1.02217 & -2.33344 \\
1.14 & 1.01751 & -2.34227 \\
1.15 & 1.01395 & -2.35055 \\
1.16 & 1.01146 & -2.35844 \\
1.17 & 1.00995 & -2.36608 \\
1.18 & 1.00939 & -2.37355 \\
1.19 & 1.00970 & -2.38092 \\
1.20 & 1.01082 & -2.38820 \\
\hline
\end{tabular}

\section{Results and discussion}

The friction factor equation (Eq. (11)) for turbulent flow in rod bundles in smooth surface is mathematically equivalent to that obtained by Lee (1995), though in a different form. Lee (1995) has shown that the friction factors predicted by this method are in good agreement with the available experimental data. We calculated the geometrical parameters, $\alpha_{1}$ and $\alpha_{2}$, in the friction factor equation, Eq. (11), for eleven pitch-to-diameter ratios in the range of $1.10-1.20$. The values of the parameters are shown in Table 2 for hexagonal arrays and in Table 3 for square arrays. With the calculated values, the friction factor equation (Eq. (11)) can be used in the same way as the Karman-Nikuradse equation for the friction factor in a circular pipe. We see in the Tables 2 and 3 that the values of $\alpha_{1}$ are near unity and approach to unity with increasing pitch-to-diameter ratio, while the values of $\alpha_{2}$ are quite different from the value in the equivalent circular pipe equation, - 1.5. It is also interesting to compare Eq. (11) with the friction factor equation by Rehme (1973).

In Figs. 2 and 3, we show the prediction by the proposed friction factor equation compared with that by the well-known correlation by Cheng and Todreas (1985). It can be seen that our analytical prediction is in good agreement with the empirical correlation based on a large pressure drop data base; the agreement is better for higher Reynolds numbers. The analytical prediction must necessarily be slightly in error, particularly for lower Reynolds numbers, since the laminar viscous layer is not taken into account, in the law of the wall used. Comparing Figs. 2 and 3, we see that the agreement is slightly better for $P / D=1.20$ than for $P / D=1.05$.

In Tables 4 and 5 , we present the calculated values of the coefficients, $\beta_{0}, \beta_{1}, \beta_{2}, \gamma_{1}$ and $\gamma_{2}$ the Nusselt number equation, Eq. (22), for eleven pitch-to-diameter ratios in the range of 1.10-1.20, for hexagonal and square rod bundles, respectively. The coefficients are not purely geometrical, as the constants in the laws of the wall for velocity and for temperature are used in the calculation $\left(\varkappa=0.4 ; B=5.5\right.$, and $\left.\chi_{\mathrm{t}}=0.44\right)$. We leave the 


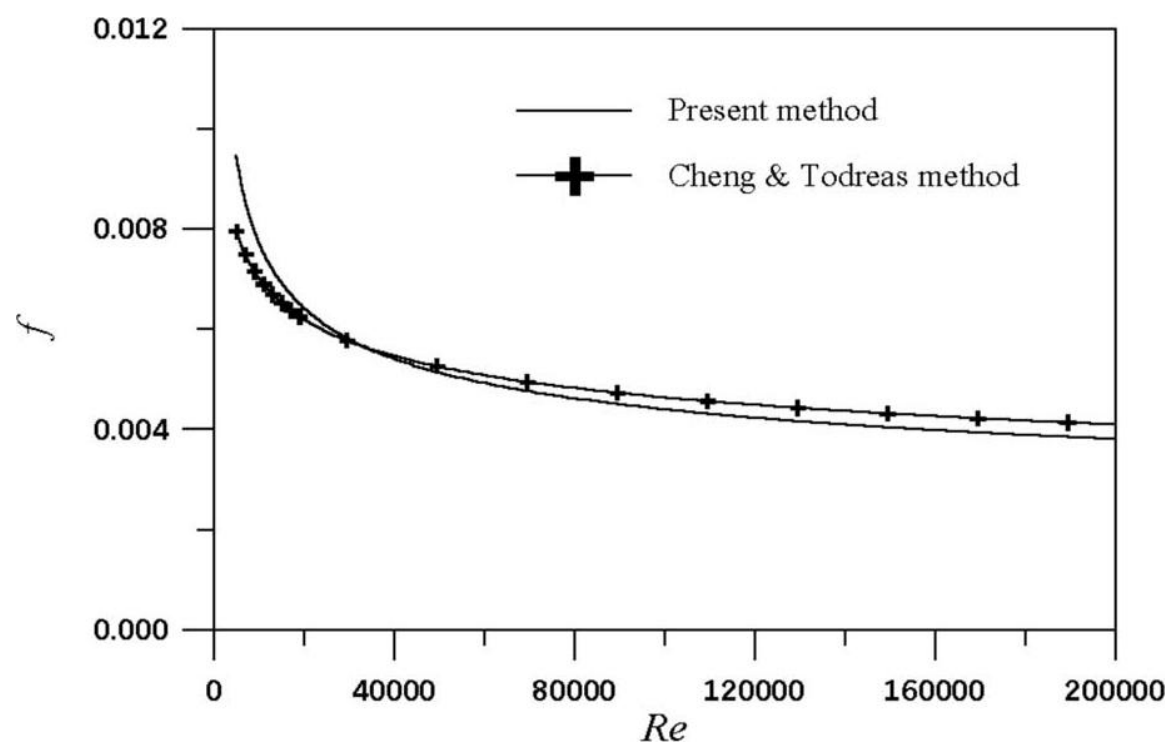

Fig. 2. Friction factor for an hexagonal array of smooth rods, $P / D=1.05$.

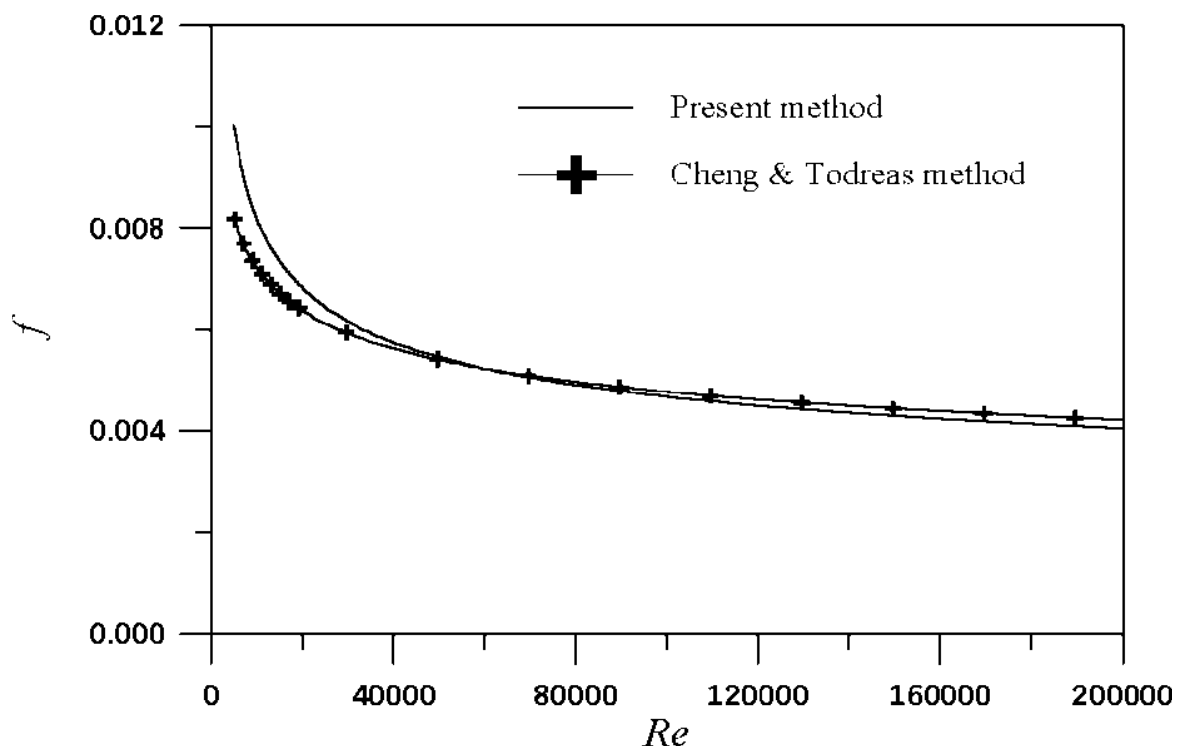

Fig. 3. Friction factor for an hexagonal array of smooth rods, $P / D=1.20$.

Prandtl number dependent coefficient $B_{\mathrm{t}}(\mathrm{Pr})$ explicit in the Nusselt number equation so that the coefficients are independent of the Prandtl number. If other sets of the constants are used, the coefficients $\beta_{0}, \beta_{1}, \beta_{2}, \gamma_{1}$ and $\gamma_{2}$ can be calculated by using the expressions given in the Appendix B.
In Figs. 4 and 5, we compare Nusselt number calculated by Eq. (22) with that by well-known correlations for Nusselt numbers in rod bundles (Presser, 1967; Weisman, 1959). It can be seen, for both square and hexagonal arrays, that the present method gives predictions similar to those 
of correlations (Presser, 1967; Weisman, 1959). In the case of Nusselt number for a hexagonal array, the curve given by the present method is graphically indistinguishable from the curve given by Presser (1967).

For the turbulent flow in rod bundles with rough surface, the transcendental equation Eq. (26) is solved numerically to obtain the friction factor, $f$, as a function of the Reynolds number, $R e$, the pitch-to-diameter ratio $P / D$, and the average roughness height $k / D$. A typical result is shown in Fig. 6. We can observe that the surface roughness increases considerably the friction factor. For the roughness heights considered, $k / D=$ $0.001,0.002,0.005, k^{+}$has the value of 43,113 , and 312, ranged from transitional roughness to fully rough regime. Typical results of Stanton numbers obtained from Eq. (28) are shown in Fig. 7. We see that surface roughness enhances the turbulent heat transfer in rod bundle, but less in percentage when compared with the increase in friction factor. In Fig. 8, we show the ratio of the Stanton number to that of a smooth circular pipe, $S t_{\mathrm{s}}$, for a square array of rod bundle. We can notice that for a same surface roughness, the heat transfer enhancement varies with $P / D$ and increases with increased Reynolds number. In Fig. 9, we can verify the departure from Reynolds analogy $(S t=f / 2)$ for various surface roughness. With smooth surface, the Reynolds analogy is

Table 4

Geometrical parameters in the Nusselt number equation (Eq. (22)) for smooth rod bundles in hexagonal arrays

\begin{tabular}{llllll}
\hline$P / D$ & $\beta_{0}$ & $\beta_{1}$ & $\beta_{2}$ & $\gamma_{1}$ & $\gamma_{2}$ \\
\hline 1.10 & 6.41687 & -15.8121 & 8.95706 & 2.56675 & -0.338999 \\
1.11 & 6.38158 & -15.9337 & 9.05785 & 2.55263 & -0.378844 \\
1.12 & 6.35266 & -16.0394 & 9.14601 & 2.54106 & -0.412703 \\
1.13 & 6.32914 & -16.1324 & 9.22362 & 2.53166 & -0.441658 \\
1.14 & 6.31025 & -16.2153 & 9.29233 & 2.51815 & -0.466549 \\
1.15 & 6.29537 & -16.2900 & 9.35346 & 2.51361 & -0.488035 \\
1.16 & 6.28403 & -16.3581 & 9.40809 & 2.51032 & -0.522787 \\
1.17 & 6.27581 & -16.4207 & 9.45708 & 2.50816 & -0.536813 \\
1.18 & 6.27039 & -16.4789 & 9.50115 & 2.50700 & -0.548991 \\
1.19 & 6.26749 & -16.5334 & 9.54086 & 2.50675 \\
1.20 & 6.26688 & -16.5848 & 9.57667 & -0.559541 \\
\hline
\end{tabular}

Table 5

Geometrical parameters in the Nusselt number equation (Eq. (22)) for smooth rod bundles in square arrays

\begin{tabular}{|c|c|c|c|c|c|}
\hline$P / D$ & $\beta_{0}$ & $\beta_{1}$ & $\beta_{2}$ & $\gamma_{1}$ & $\gamma_{2}$ \\
\hline 1.10 & 6.21939 & -13.2602 & 7.35981 & 2.73653 & 0.0929488 \\
\hline 1.11 & 6.10990 & -13.4783 & 7.58140 & 2.68836 & -0.00804097 \\
\hline 1.12 & 6.01960 & -13.6666 & 7.77153 & 2.64862 & -0.0931628 \\
\hline 1.13 & 5.94656 & -13.8300 & 7.93343 & 2.61649 & -0.164460 \\
\hline 1.14 & 5.88917 & -13.9728 & 8.06993 & 2.59124 & -0.223653 \\
\hline 1.15 & 5.84607 & -14.0986 & 8.18348 & 2.57227 & -0.272187 \\
\hline 1.16 & 5.81608 & -14.2102 & 8.27618 & 2.55908 & -0.311270 \\
\hline 1.17 & 5.79821 & -14.3102 & 8.3498 & 2.55121 & -0.341904 \\
\hline 1.18 & 5.79158 & -14.4002 & 8.40577 & 2.54830 & -0.364907 \\
\hline 1.19 & 5.79547 & -14.4815 & 8.44519 & 2.55001 & -0.380934 \\
\hline 1.20 & 5.80920 & -14.5552 & 8.46886 & 2.55605 & -0.390495 \\
\hline
\end{tabular}




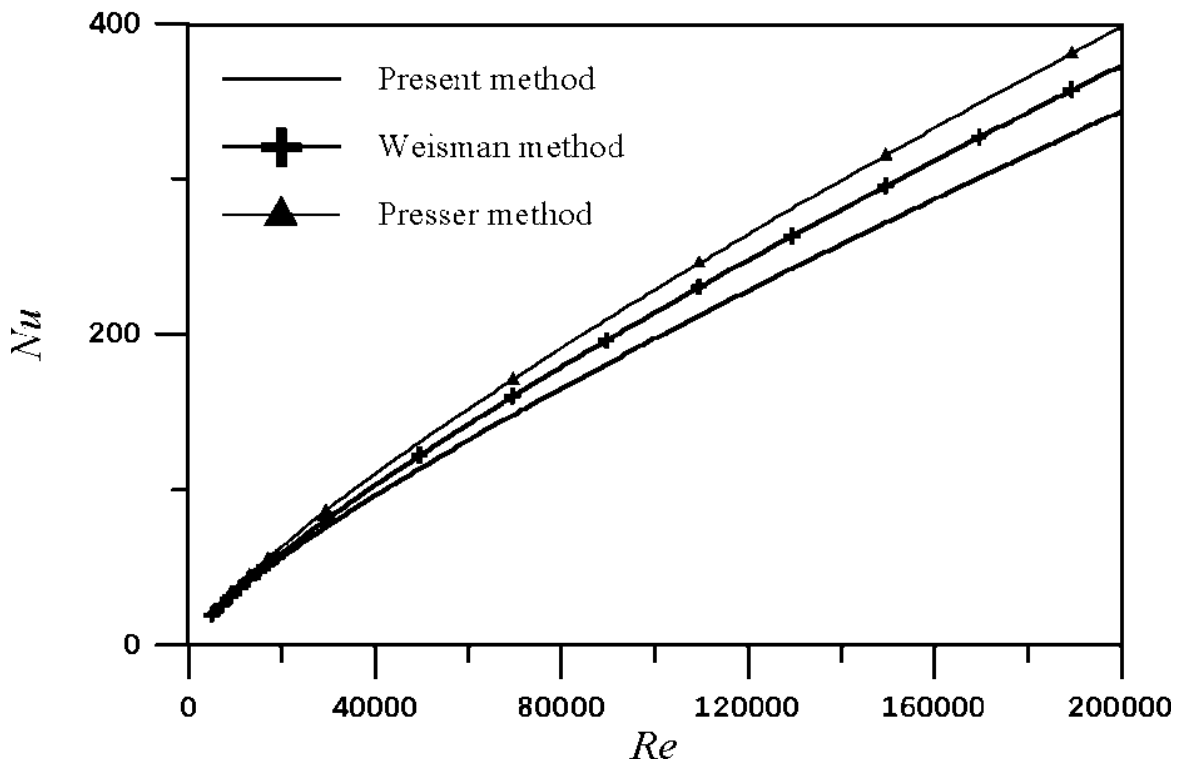

Fig. 4. Nusselt number for a square array of smooth rods, $P / D=1.1$ and $P r=0.9$.

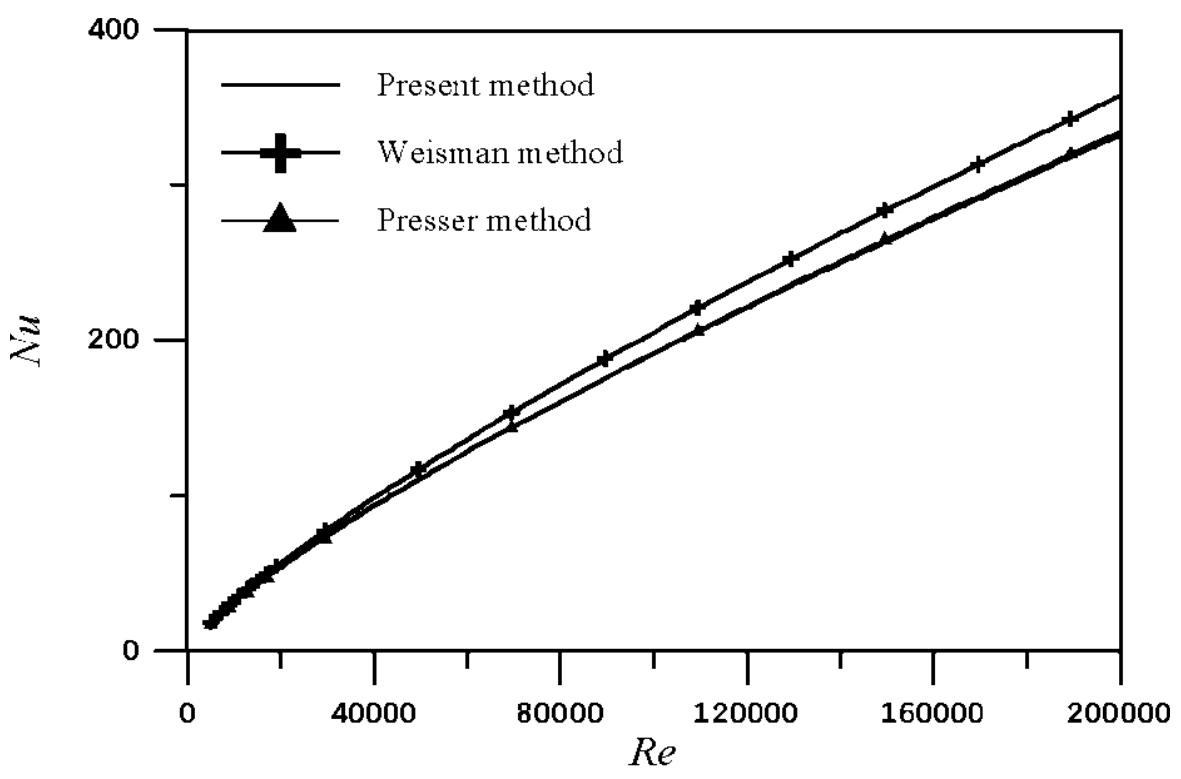

Fig. 5. Nusselt for an hexagonal array of smooth rods, $P / D=1.05$ and $P r=0.9$.

seen to be maintained, while for fully rough surface, $k / D=0.005$. St is nearly half of $f / 2$. From an engineering point of view, it would be interesting under some circumstance to adopt rod bundle heat exchanger with rough surface if it is worthy to pay the overhead in pressure drop.

\section{Conclusion}

We derived an algebraic equation for the friction factor of turbulent flow in rod bundles with smooth surface, following the work of Lee (1995). The friction factor equation was cast in a similar 
form to the classical Karman-Nikuradse equation for the friction factor of turbulent flow in smooth circular tube (Todreas and Kazimi, 1990). Two geometrical parameters, $\alpha_{1}$ and $\alpha_{2}$ are in- volved in the friction factor equation, which are determined by the type of arrangement and the pitch-to-diameter ratio $(P / D)$. For a specific rod bundle, $\alpha_{1}$ and $\alpha_{2}$ are fixed; the friction factor

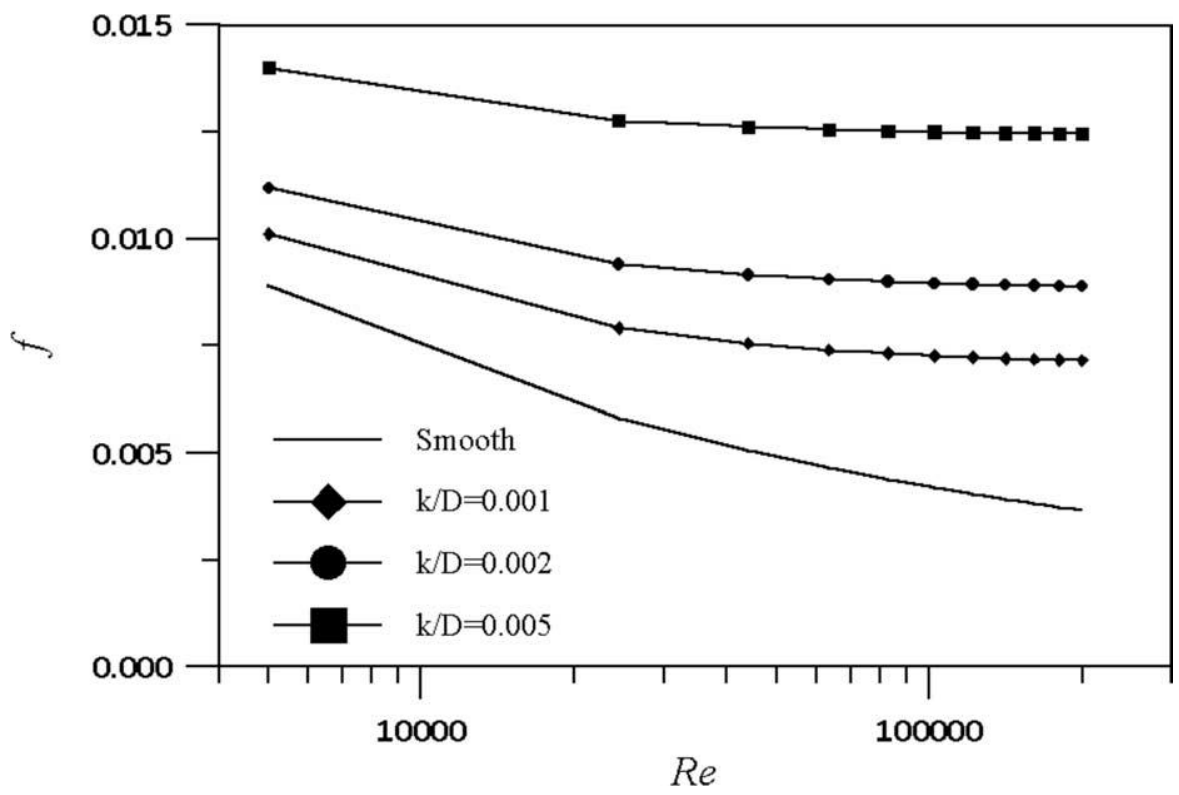

Fig. 6. Friction factor for an hexagonal array, $P / D=1.05$.

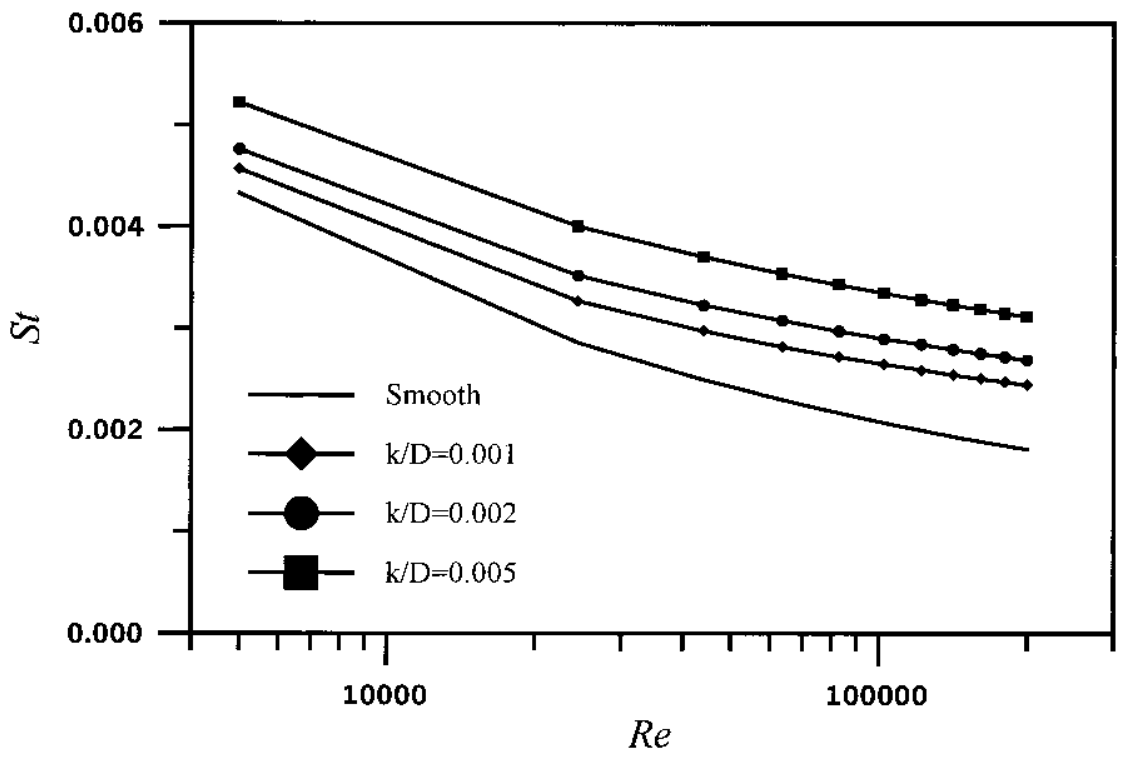

Fig. 7. Stanton numbers for an hexagonal array, $P / D=1.05$ and $\operatorname{Pr}=0.9$. 


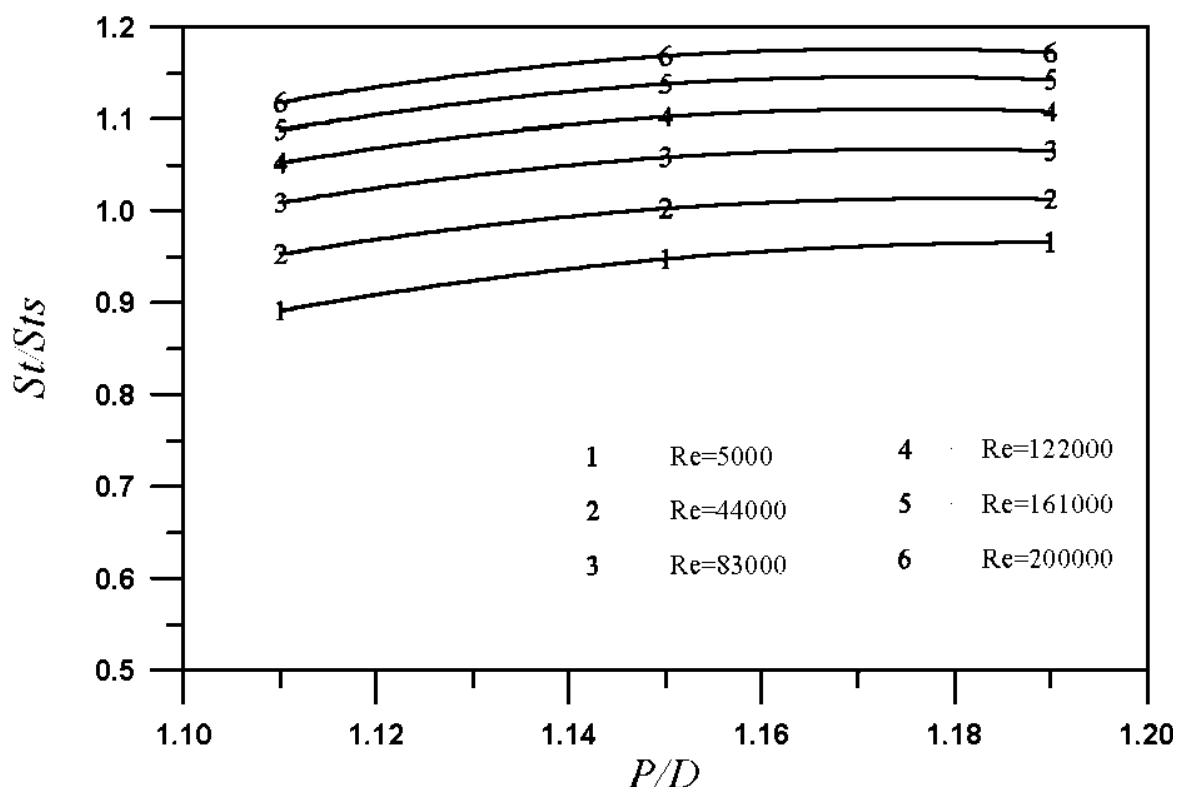

Fig. 8. Influence of pitch-to-diameter ratio and Reynolds number on Stanton numbers: square array, $k / D=0.001$ and $P r=0.9$.

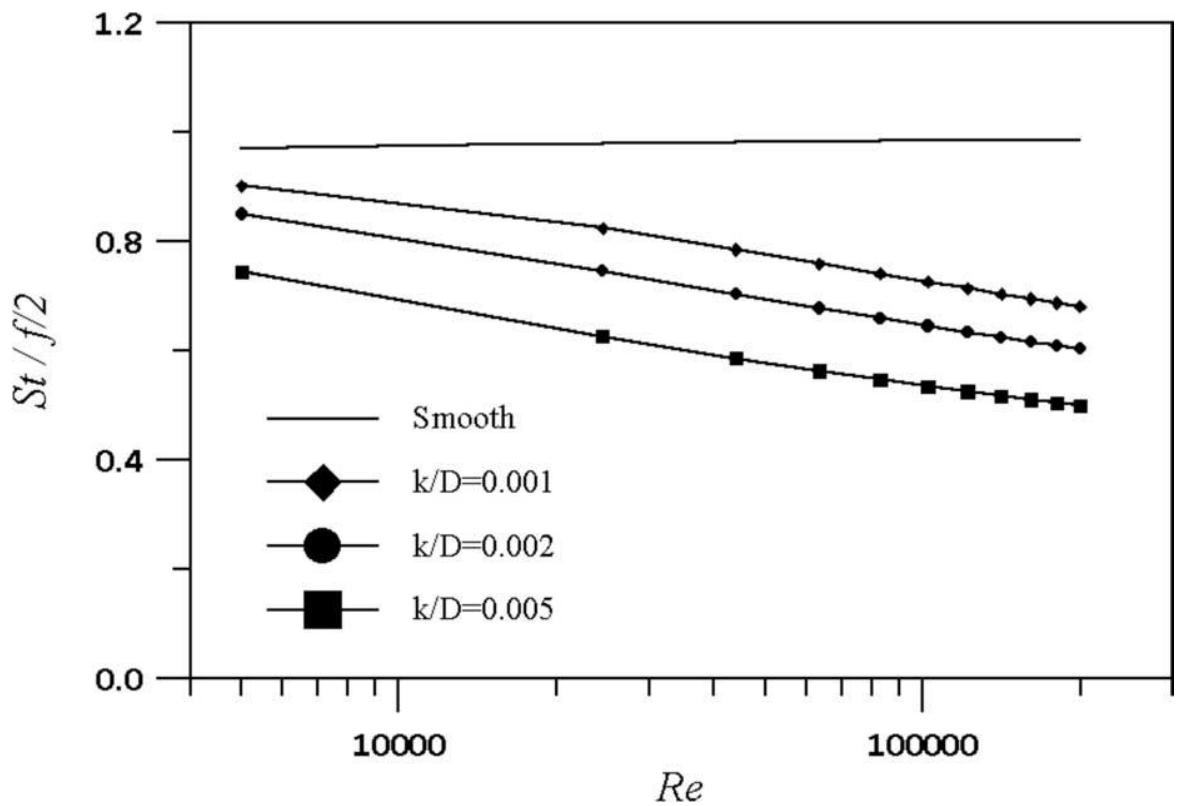

Fig. 9. Departure from Reynolds analogy: hexagonal array, $P / D=1.05$ and $P r=0.9$.

equation can be used in the same way as the Karman-Nikuradse equation. Although mathematically the friction factor equation presented here is the same as presented by Lee (1995), it is in a form that is more convenient to use in engineering applications. It is structurally similar to the friction factor equation proposed by Rehme (1973). We can observe from the develop- 
ment of the friction factor equation that the geometry of the flow channel is represented by $\theta_{\max }$ and $P / D$, and the empirical function $F(\theta)$ which is assumed to be independent of the Reynolds number. After integration, all geometrical informations are represented by the two parameters $\alpha_{1}$ and $\alpha_{2}$ which we made intentionally independent of the constants $\varkappa$ and $B$ of the law of the wall for velocity.

We derived an explicit equation for the Nusselt number of turbulent convection in rod bundles with smooth surface, as a function of the friction factor, the Reynolds number and the Prandtl number. As Churchill (2001) stated, the derived Nusselt number was not a fixed power function of Reynolds and Prandtl numbers. Five coefficients appeared in the expression, which involved the universal constants $\varkappa, B, \varkappa_{\mathrm{t}}$, and geometrical parameters $P / D$ and $\theta_{\max }$. If universal constants are adopted for $\varkappa, B, \varkappa_{t}$, the coefficients are fixed for a given rod bundle. The proposed Nusselt equation was as simple as the empirical correlations in literature, but with a sound physical foundation. It might in some way clarify the complex phenomenon of turbulent convection in rod bundles by identifying the functional relation among the key parameters.

We applied the same analytical method to predict the friction factor of turbulent flow and the Nusselt number of turbulent forced convection in rod bundles with rough surface. In this case, numerical integration was necessary to obtain the friction factor and Nusselt number. Though the proposed method is not as convenient as the explicit algebraic expression for smooth surface, it provided a useful, predictive engineering tool, and some understanding of the physics involved in the phenomena.

\section{Acknowledgements}

The authors acknowledge the Brazilian National Research Council (CNPq) for the financial support during the realisation of this work. J. Su is grateful to Professor G.F. Hewitt and the Department of Chemical Engineering and Chemical Technology of Imperial College of Science, Tech- nology and Medicine for the hospitality during his academic visit, supported by the Ministry of Education of Brazil (MEC/CAPES) through a scholarship.

\section{Appendix A. Nomenclature}

$A$ flow area of channel; geometry factor in Eq. (1)

$B$ constant $(=5.0)$

$B_{\mathrm{t}} \quad$ function of the Prandtl number

$a, b$ constants

$c_{\mathrm{p}} \quad$ specific heat of the fluid

$D$ rod diameter

$D_{\mathrm{H}} \quad$ hydraulic diameter

$d_{\mathrm{h}} \quad$ dimensionless hydraulic diameter

$F(\theta)$ function of $\theta$

$f \quad$ Fanning friction factor

$H(\theta)$ function of $\theta$

$h$ heat transfer coefficient

$I \quad$ integrals given in the Appendix B

$J \quad$ integrals given in the Appendix B

$k \quad$ average height of surface roughness

$k^{+} \quad$ dimensionless surface roughness

$k_{\mathrm{s}} \quad$ length scale of surface roughness

$\mathrm{Nu} \quad$ Nusselt number

$P \quad$ pitch of rod array

$p \quad$ pitch-to-rod diameter ratio $P / D$

Pr Prandtl number

$q_{\mathrm{w}} \quad$ wall heat flux

$\bar{q}_{\mathrm{w}} \quad$ mean wall heat flux

$R \quad$ radius of the rod

St Stanton number

$S t_{\text {s }} \quad$ Stanton number of a smooth circular pipe

$T$ temperature

$\bar{T} \quad$ mean temperature

$T_{\mathrm{w}} \quad$ wall temperature

$\bar{T}_{\mathrm{w}} \quad$ mean wall temperature

$T_{\tau} \quad$ local friction temperature

$\frac{T_{\tau}}{T_{\tau}} \quad$ mean friction temperature

$u \quad$ flow velocity

$\bar{u} \quad$ average flow velocity

$u_{\tau} \quad$ local friction velocity

$\bar{u}_{\tau} \quad$ average friction velocity

$y$ normal distance from wall

$y^{+}$dimensionless wall distance 
Greek letters

$\alpha_{0}, \alpha_{1}, \alpha_{2}$ geometrical parameters given in the Appendix B

$\beta_{0}, \beta_{1}, \beta_{2}$ geometrical parameters given in the Appendix B

$\gamma_{1}, \gamma_{2} \quad$ geometrical parameters given in the Appendix B

$\theta \quad$ angular position around the rod

$\varkappa \quad$ von Karman's constant $(=0.4)$

$x_{\mathrm{t}} \quad$ constant $(=0.44)$

$\rho \quad$ density of fluid

$\lambda \quad$ thermal conductivity of the fluid;

Darcy friction factor in Eq. (1)

$\mu \quad$ viscosity of fluid

$v \quad$ kinematic viscosity of fluid

$\tau_{\mathrm{w}} \quad$ local wall shear stress

$\bar{\tau}_{\mathrm{w}} \quad$ average wall shear stress

\section{Appendix B}

We give here the details of the development of the friction factor and Nusselt number equations for smooth surface bundles and the definitions of the coefficients that appear in the equations.

The integration in $y$ can be done analytically:

$$
\begin{aligned}
I_{0}(\theta) & =\frac{1}{R^{2}} \int_{0}^{(P / 2 \sec \theta-R)}(R+y) \mathrm{d} y \\
& =\frac{1}{2 R^{2}}\left(\frac{P}{2} \sec \theta-R\right)\left(\frac{P}{2} \sec \theta+R\right) \\
& =\frac{1}{2}(p \sec \theta-1)(p \sec \theta+1)
\end{aligned}
$$

where $p(=P / D)$ denotes the pitch-to-diameter ratio.

$$
\begin{aligned}
I_{1}(\theta) & =\frac{1}{R^{2}} \int_{0}^{(P / 2 \sec \theta-R)} \ln \left(\frac{y}{D_{\mathrm{H}}}\right)(R+y) \mathrm{d} y \\
& =\frac{1}{R^{2}}\left[\frac{1}{2}\left(\frac{P}{2} \sec \theta-R\right)\left(\frac{P}{2} \sec \theta-R\right)\right.
\end{aligned}
$$

$$
\begin{aligned}
\times & \ln \left(\frac{\frac{P}{2} \sec \theta-R}{D_{\mathrm{H}}}\right) \\
& \left.-\frac{1}{4}\left(\frac{P}{2} \sec \theta-R\right)\left(\frac{P}{2} \sec \theta+3 R\right)\right] \\
= & {\left[\frac{1}{2}(p \sec \theta-1)(p \sec \theta+1)\right.} \\
& \times \ln \left(\frac{(p \sec \theta-1)}{2 d_{\mathrm{h}}}\right) \\
& \left.-\frac{1}{4}(p \sec \theta-1)(p \sec \theta+3)\right]
\end{aligned}
$$

where $d_{\mathrm{h}}\left(D_{\mathrm{H}} / D\right)$ is the dimensionless hydraulic diameter.

$$
\begin{aligned}
& I_{2}(\theta)=\frac{1}{R^{2}} \int_{0}^{(P / 2 \sec \theta-R)}\left[\ln \left(\frac{y}{D_{\mathrm{H}}}\right)\right]^{2}(R+y) \mathrm{d} y \\
& =\frac{1}{R^{2}}\left[2 R\left(-R+\frac{P \sec \theta}{2}\right)\right. \\
& +\frac{1}{4}\left(-R+\frac{P \sec \theta}{2}\right)^{2} \\
& +\frac{1}{2} \log \left(\frac{-R+(P \sec \theta / 2)}{D_{\mathrm{h}}}\right)^{2} \\
& \times\left(-R+\frac{P \sec \theta}{2}\right)\left(R+\frac{P \sec \theta}{2}\right) \\
& -\frac{1}{2} \log \left(\frac{-R+(P \sec \theta / 2)}{D_{\mathrm{h}}}\right) \\
& \left.\times\left(-R+\frac{P \sec \theta}{2}\right)\left(3 R+\frac{P \sec \theta}{2}\right)\right] \\
& =\left[2(-1+p \sec \theta)+\frac{1}{4}(-1+p \sec \theta)^{2}\right. \\
& +\frac{1}{2} \log \left(\frac{-p \sec \theta}{2 d_{\mathrm{h}}}\right)^{2}(-1+p \sec \theta) \\
& \times(1+p \sec \theta) \\
& -\frac{1}{2} \log \left(\frac{-1+p \sec \theta}{2 d_{\mathrm{h}}}\right)(-1+p \sec \theta) \\
& \times(3+p \sec \theta)]
\end{aligned}
$$

The area of the flow channel is 


$$
\begin{aligned}
A & =\int_{0}^{\theta_{\max }} \int_{0}^{(P / 2 \sec \theta-R)}(R+y) \mathrm{d} y \mathrm{~d} \theta \\
& =R^{2} \int_{0}^{\theta_{\max }} I_{0}(\theta) \mathrm{d} \theta=R^{2} \alpha_{0}
\end{aligned}
$$

where

$$
\alpha_{0}=\int_{0}^{\theta_{\max }} I_{0}(\theta) \mathrm{d} \theta
$$

The development of the equation for the friction velocity, Eq. (10), is given by

$$
\begin{aligned}
& \frac{\bar{u}}{\bar{u}_{\tau}}=\frac{1}{A} \int_{0}^{\theta_{\max }} \int_{0}^{(P / 2 \sec \theta-R)}\left(\frac{1}{\chi} \ln \frac{y \bar{u}_{\tau} F(\theta)}{v}+B\right) F(\theta) \\
& \times(R+y) \mathrm{d} y \mathrm{~d} \theta \\
& =\frac{1}{A} \int_{0}^{\theta_{\max }} \int_{0}^{(P / 2 \sec \theta-R)}\left(\frac{1}{\chi} \ln \frac{D_{\mathrm{H}} \bar{u}_{\tau}}{v}+B\right. \\
& \left.+\frac{1}{x} \ln F(\theta)+\frac{1}{x} \ln \frac{y}{D_{\mathrm{H}}}\right) F(\theta)(R+y) \mathrm{d} y \mathrm{~d} \theta \\
& =\frac{1}{A} \int_{0}^{\theta_{\max }}\left(\frac{1}{\varkappa} \ln \frac{D_{\mathrm{H}} \bar{u}_{\tau}}{v}+B+\frac{1}{\chi} \ln F(\theta)\right) F(\theta) \\
& \times \int_{0}^{(P / 2 \sec \theta-R)}(R+y) \mathrm{d} y \mathrm{~d} \theta \\
& +\frac{1}{A} \int_{0}^{\theta_{\max }} \frac{1}{\chi} F(\theta) \\
& \times \int_{0}^{(P / 2 \sec \theta-R)} \int_{0}^{(P / 2 \sec \theta-R)} \ln \frac{y}{D_{\mathrm{H}}}(R+y) \\
& \times \mathrm{d} y \mathrm{~d} \theta=\frac{1}{\alpha_{0}} \int_{0}^{\theta_{\max }}\left(\frac{1}{x} \ln \frac{D_{\mathrm{H}} \bar{u}_{\tau}}{v}+B\right. \\
& \left.+\frac{1}{\varkappa} \ln F(\theta)\right) F(\theta) I_{0}(\theta) \mathrm{d} \theta \\
& +\frac{1}{\alpha_{0}} \int_{0}^{\theta_{\max }} \frac{1}{\varkappa} F(\theta) I_{1}(\theta) \mathrm{d} \theta \\
& =\frac{1}{\alpha_{0}}\left(\frac{1}{\chi} \ln \frac{D_{\mathrm{H}} \bar{u} \tau}{v}+B\right) \int_{0}^{\theta_{\max }} F(\theta) I_{0}(\theta) \mathrm{d} \theta \\
& +\frac{1}{\alpha_{0}} \frac{1}{\varkappa} \int_{0}^{\theta_{\max }}\left(\ln F(\theta) I_{0}(\theta)+I_{1}(\theta)\right) F(\theta) \mathrm{d} \theta,
\end{aligned}
$$

which results in

$$
\frac{\bar{u}}{\bar{u}_{\tau}}=\left(\frac{1}{x} \ln \frac{D_{\mathrm{H}} \bar{u}_{\tau}}{v}+B\right) \alpha_{1}+\frac{\alpha_{2}}{\chi}
$$

where

$$
\begin{aligned}
& \alpha_{1}=\frac{1}{\alpha_{0}} \int_{0}^{\theta_{\max }} F(\theta) I_{0}(\theta) \mathrm{d} \theta \\
& \alpha_{2}=\frac{1}{\alpha_{0}} \int_{0}^{\theta_{\max }}\left(\ln F(\theta) I_{0}(\theta)+I_{1}(\theta)\right) F(\theta) \mathrm{d} \theta
\end{aligned}
$$
by

The equation for the Stanton number is given $\frac{\bar{u}\left(\bar{T}_{\mathrm{w}}-\bar{T}\right)}{\bar{u}_{\tau} \bar{T}_{\tau}}=\frac{1}{A} \int_{0}^{\theta_{\max }} \int_{0}^{(P / 2 \sec \theta-R)}\left(\frac{1}{\chi} \ln \frac{y \bar{u}_{\tau} F(\theta)}{v}\right.$

$$
\begin{aligned}
& +B)\left(\frac{1}{\varkappa_{\mathrm{t}}} \ln \frac{y \bar{u}_{\tau} F(\theta)}{v}+B_{\mathrm{t}}(P r)\right) F(\theta) \\
& \times H(\theta)(R+y) \mathrm{d} y \mathrm{~d} \theta \\
& =\left(\frac{1}{x} \ln \frac{D_{\mathrm{H}} \bar{u}_{\tau}}{v}+B\right)\left(\frac{1}{x_{\mathrm{t}}} \ln \frac{D_{\mathrm{H}} \bar{u}_{\tau}}{v}+B_{\mathrm{t}}\right) J_{0} \\
& +\left(\frac{2}{\varkappa \varkappa_{\mathrm{t}}} \ln \frac{D_{\mathrm{H}} \bar{u}_{\tau}}{v}+\frac{B}{\varkappa_{\mathrm{t}}}+\frac{B_{\mathrm{t}}}{\varkappa}\right) J_{1}+\frac{1}{\varkappa \varkappa_{\mathrm{t}}} J_{2} \\
& +\left(\frac{2}{\varkappa \varkappa_{\mathrm{t}}} \ln \frac{D_{\mathrm{H}} \bar{u}_{\tau}}{v}+\frac{B}{\chi_{\mathrm{t}}}+\frac{B_{\mathrm{t}}}{\varkappa}\right) J_{3}+\frac{2}{\varkappa \varkappa_{\mathrm{t}}} J_{4} \\
& +\frac{1}{x x_{t}} J_{5}
\end{aligned}
$$

$J_{0}=\frac{1}{\alpha_{0}} \int_{0}^{\theta_{\max }} I_{0}(\theta) F(\theta) H(\theta) \mathrm{d} \theta$

$J_{1}=\frac{1}{\alpha_{0}} \int_{0}^{\theta_{\max }} I_{0}(\theta) \ln (F(\theta)) F(\theta) H(\theta) \mathrm{d} \theta$

$J_{2}=\frac{1}{\alpha_{0}} \int_{0}^{\theta_{\max }} I_{0}(\theta)(\ln (F(\theta)))^{2} F(\theta) H(\theta) \mathrm{d} \theta$

$J_{3}=\frac{1}{\alpha_{0}} \int_{0}^{\theta_{\max }} I_{1}(\theta) F(\theta) H(\theta) \mathrm{d} \theta$

$J_{4}=\frac{1}{\alpha_{0}} \int_{0}^{\theta_{\max }} I_{1}(\theta) \ln (F(\theta)) F(\theta) H(\theta) \mathrm{d} \theta$

$J_{5}=\frac{1}{\alpha_{0}} \int_{0}^{\theta_{\max }} I_{2}(\theta) F(\theta) H(\theta) \mathrm{d} \theta$

Thus, the equation can be written as

$$
\begin{aligned}
\frac{1}{S t}= & \beta_{0}\left(\ln \left[\operatorname{Re}\left(\frac{f}{2}\right)^{1 / 2}\right]\right)^{2} \\
& +\left(\beta_{1}+\gamma_{1} B_{\mathrm{t}}(\operatorname{Pr})\right) \ln \left[\operatorname{Re}\left(\frac{f}{2}\right)^{1 / 2}\right]+\beta_{2} \\
& +\gamma_{2} B_{\mathrm{t}}(\operatorname{Pr})
\end{aligned}
$$


where

$\beta_{0}=\frac{J_{0}}{x x_{\mathrm{t}}}$

$\beta_{1}=\frac{2}{x_{\mathrm{t}}}\left(J_{1}+J_{3}\right)+\frac{J_{0} B}{\varkappa_{\mathrm{t}}}$

$\gamma_{1}=\frac{J_{0}}{\varkappa}$

$\beta_{2}=\frac{J_{2}+2 J_{4}+J_{5}}{\varkappa \varkappa_{\mathrm{t}}}+\frac{B}{\varkappa_{\mathrm{t}}}\left(J_{1}+J_{3}\right)$

$\gamma_{2}=B J_{0}+\frac{J_{1}+J_{3}}{\varkappa}$

\section{References}

Bartzis, J.G., Todreas, N.E., 1979. Turbulence modelling of axial flow in a bare rod bundle. ASME J. Heat Transfer 101, 628-634.

Carajileskov, P., Todreas, N.E., 1976. Experimental and analytical study of axial turbulent flows in an interior subchannel of a bare rod bundle. ASME J. Heat Transfer 98, 262-268.

Cheng, S.K., Todreas, N.E., 1985. Hydrodynamic models and correlations for wire-wrapped LMFBR bundles and subchannel friction factors and mixing parameters. Nucl. Eng. Des. 92, 227-251.

Chun, M.-H., Seo, K.-W., 2001. An experimental study and assessment of existing friction factor correlations for wirewrapped fuel assemblies. Ann. Nucl. Energy 28, 16831695.

Churchill, S.W., 2001. Turbulent flow and convection: the prediction of turbulent flow and convection in a round tube. Adv. Heat Transfer 34, 255-361.

Clauser, F.H., 1956. The turbulent boundary layer. Adv. Appl. Mech. 4, 1-51.

Fakory, M., Todreas, N.E., 1979. Experimental investigation of flow resistance and wall shear stress in the interior subchannel of a triangular array of parallel rods. ASME J. Fluids Eng. 101, 429-435.

Hooper, J.D., 1980. Developed single phase turbulent flow through a square-pitch rod cluster. Nucl. Eng. Des. 60, 365-379.

Hooper, J.D., Rehme, K., 1984. Large-scale structural effects in developed turbulent flow through closely-spaced rod arrays. J. Fluid Mech. 145, 305-337.

Hooper, J.D., Wood, D.H., 1984. Fully developed rod bundle flow over a large range of Reynolds number. Nucl. Eng. Des. 83, 31-46.
Kim, N.-H., Lee, S.-K., Kim, S.-H., 1993. Development of an algebraic model to predict the heat transfer for turbulent flow in a rod bundle. ASME J. Heat Transfer 115, 266268.

Krauss, T., Meyer, L., 1996. Characteristics of turbulent velocity and temperature in a wall channel of a heated rod bundle. Exp. Thermal Fluid Sci. 12, 75-86.

Krauss, T., Meyer, L., 1998. Experimental investigation of turbulent transport of momentum and energy in a heated rod bundle. Nucl. Eng. Des. 180, 185-206.

Lee, K.B., 1995. Analytical prediction of subchannel friction factor for infinite bare rod square and triangular arrays of low pitch-to-diameter ratio in turbulent flow. Nucl. Eng. Des. 157, 197-203.

Marek, J., Maubach, K., Rehme, K., 1973. Heat transfer and pressure drop performance of rod bundles arranged in square arrays. Int. J. Heat Mass Transfer 16, 2215-2228.

Meyer, L., 1994. Measurements of turbulent velocity and temperature in axial flow through a heated rod bundle. Nucl. Eng. Des. 146, 71-82.

Möller, S.V., 1991. On phenomena of turbulent-flow through rod bundles. Exp. Thermal Fluid Sci. 4, 25-35.

Möller, S.V., 1992. Single-phase turbulent mixing through rod bundles. Exp. Thermal Fluid Sci. 5, 26-33.

Nikuradse, J., 1933. Stromungsgesetze in rauhen rohren. V.D.I. Forshungsheft No 361.

Presser, K.H., 1967. Warmeubergang und druckerlust and reaktorbrennelementen in form langsdurchstromter rundstabbundel. Jul-484-RB, KFA, Julich.

Rehme, K., 1972. Pressure drop performance of rod bundles in hexagonal arrangements. Int. J. Heat Mass Transfer 15, 2499-2517.

Rehme, K., 1973. Simple method of predicting friction factors of turbulent flow in a non-circular channel. Int. J. Heat Mass Transfer 16, 933-950.

Rehme, K., 1987. The structure of turbulent flow through rod bundles. Nucl. Eng. Des. 99, 141-154.

Rehme, K., 1992. The structure of turbulence in rod bundles and the implications on natural mixing between the subchannels. Int. J. Heat Mass Transfer 35, 567-581.

Renksizbulut, M., Hadaller, G.I., 1986. An experimental study of turbulent flow through a square-array rod bundle. Nucl. Eng. Des. 91, 41-55.

Todreas, N.E., Kazimi, M.S., 1990. Nuclear Systems I. Hemisphere, New York.

Trupp, A.C., Azad, R.S., 1975. The structure of turbulent flow in triangular array rod bundles. Nucl. Eng. Des. 32, 47-84.

Vijayan, P.K., Pikhwal, D.S., Saha, D., Venkat Raj, V., 1999. Experimental study on the pressure drop across the various components of a PHWR fuel channel. Exp. Thermal Fluid Sci. 20, 34-44.

Weisman, J., 1959. Heat transfer to water flowing parallel to tube bundles. Nucl. Eng. Des. 6, 78-79.

White, F.M., 1974. Viscous Fluid Flow. McGraw-Hill, New York. 